\title{
Valuación de opciones asiáticas con precio de ejercicio flotante igual a la media aritmética: un enfoque de control óptimo estocástico María Teresa Verónica Martínez-Palacios * Instituto Politécnico Nacional, Escuela Superior de Economía Ambrosio Ortiz-Ramírez \\ Instituto Politécnico Nacional, Escuela Superior de Economía, José Francisco Martínez-Sánchez \\ Universidad Autónoma Del Estado de Hidalgo, Escuela Superior de Apan
}

(Primera recepción: 24 de Mayo 2016. Segunda recepción: 30 de Octubre 2016. Aceptado 26 de Marzo 2016.)

\section{Resumen}

En esta investigación se caracteriza la prima de una opción de venta asiática de tipo europeo con precio de ejercicio flotante igual a la media aritmética, mediante el análisis de solución a un problema de control óptimo estocástico en tiempo continuo, que modela el proceso de toma de decisiones de consumo-inversión de un consumidor racional en un horizonte finito de planeación con fecha final estocástica. La prima obtenida es una ecuación diferencial parcial de segundo orden que corresponde a la ecuación de Black-Scholes-Merton, con la diferencia de que esta se establece con fundamentos de racionalidad económica. Asimismo, se resuelve analíticamente la ecuación diferencial parcial de Hamilton-Jacobi-Bellman que optimiza el problema de control óptimo estocástico planteado.

Clasificación: C02, D91, G12, D81.

Palabras clave: Control óptimo estocástico en tiempo continuo, Decisiones de consumo y portafolio, Opciones asiáticas, modelos de equilibrio

\section{Pricing Asian Options with Floating Exercise Price Equal to the Arithmetic Mean: An Optimal Stochastic Control Approach}

\begin{abstract}
This research characterizes the premium of a European-type +Asian put option with floating exercise price equal to the arithmetic mean, through the solution analysis to a stochastic optimal control problem in continuous time, that models the decision-making process of consumption-investment of a rational consumer in a finite horizon of planning with stochastic terminal date. The premium obtained is a partial differential equation of second order corresponding to the Black-Scholes-Merton equation, with the difference that this is established with fundamentals of economic rationality. Also, the Hamilton-Jacobi-Bellman partial differential equation is solved, which optimizes the stochastic optimal control problem.

JEL Classification: C02, D91, G12, D81.

Key Words: Optimal Stochastic Control,Problem, Consumption and Portfolio Decisions, Asian Options, Equilibrium Models.
\end{abstract}

* Escuela Superior de Economía-I. P. N., Unidad Profesional Lázaro Cárdenas, Plan de Agua Prieta No. 66, Col. Plutarco Elías Calles, Delegación Miguel Hidalgo, C.P. 11340, México, D.F. Tel. 57296000 ext. 62036. 


\section{Introducción}

Las características fehacientes del riesgo inherente a los mercados financieros, en que los diversos agentes económicos realizan transacciones de manera "continua" con activos y/o productos derivados financieros, influye en las decisiones de consumo, portafolio y producción de los agentes económicos.

En este contexto, las necesidades específicas de cobertura de riesgos, fiscales, contables, legales o regulatorias, de los diferentes agentes económicos (Hull, 2014) y de adaptación de las características de los diferentes instrumentos derivados a tales requerimientos, ha hecho de la ingeniería financiera un campo fértil de innovación en los mercados secundarios o mercados Over The Counter (OTC).

Por su parte, la comunidad académica internacional de diversas áreas como lo son: Economía, Finanzas, Matemáticas e Ingeniería, entre otras, en respuesta a tales necesidades ha desarrollado diversos modelos en los que se supone que las variables económicas y financieras relevantes son conducidas por procesos estocásticos de diferente naturaleza, por ejemplo, procesos de difusión o procesos de difusión con saltos como en Merton (1971) y (1992), Turnovsky (1993) y (2006), Hernández-Lerma (1994) y Venegas-Martínez (2001) y (2006).

Un aspecto importante es el desarrollo de modelos macroeconómicos estocásticos que expliquen hechos estilizados en las decisiones de consumo y portafolio de los agentes; véanse, al respecto: Turnovsky y Smith (2006); Venegas-Martínez, Ortiz-Arango y Castillo-Ramírez (2010); Martínez-Palacios, T., A. Sánchez-Daza, y F. Venegas-Martínez, (2014); Martínez-Palacios, T. y F. Venegas-Martínez, (2015); y Venegas-Martínez (2004) y (2005), entre otros.

En el marco de los modelos anteriores, es importante destacar, aquellos a partir de los cuales se obtienen precios de activos financieros y/o se valúan productos derivados; cuya literatura es muy basta y variada, considérese por ejemplo los artículos, de: Black y Scholes (1973); Merton (1973); Cox y Ross (1976); Cox, Ingersoll y Ross (1985a) y (1985b); Broadie y Detemple (2004); Martínez-Palacios, T. y F. Venegas-Martínez, (2012) y (2014); VenegasMartínez (2006), (2007) y (2010); Venegas-Martínez, F. y S. Cruz-Ake, (2010); Ángeles-Castro y Venegas-Martínez (2010) y Sierra (2007), ${ }^{1}$ entre otros.

En el caso particular de la valuación de derivados financieros opciones, la innovación ha dado lugar a la creación de opciones de tipo exótico, ${ }^{2}$ las cuales suponen una modificación de alguna o varias de las características de las opciones estándar. De particular importancia en esta investigación son las opciones asiáticas ${ }^{3}$ con precio de ejercicio promedio de tipo europeo. Estas son

\footnotetext{
1 Una particularidad del trabajo de Sierra (2007) es que sus activos son modelados mediante Brownianos fraccionales.

2 Una clasificación según Hull (Hull, 2014) es: 1. Opciones compuestas u opciones sobre opciones, 2. Opciones con valor dependiente de la evolución histórica del subyacente (pathdependents), 3. Opciones condicionales y 4. Opciones sobre varios subyacentes.

3 En las opciones asiáticas se pueden distinguir las opciones asiáticas de valor promedio del subyacente y las de precio de ejercicio promedio. Estas opciones son atractivas a los inversionistas porque el costo de sus primas es menor que el de las opciones tradicionales, dado que para valorarlas hay que tener en cuenta la volatilidad de la media de los valores que
} 
opciones en las que el precio de ejercicio es una media (aritmético o geométrico) de los valores alcanzados por el activo subyacente durante toda o parte de la vida de la opción.

La finalidad fundamental de este tipo de opciones es reducir las posibilidades de manipulación del precio del subyacente en la fecha de vencimiento. También algunos inversores las consideran útiles cuando su política de compras (o ventas) les obliga a realizar transacciones frecuentes sobre un mismo activo en un horizonte temporal determinado (Lamothe y Pérez, 2003).

La investigación que se ha dedicado al estudio de esta opción es también muy extensa, toda vez que no existe una solución analítica cerrada para obtener el precio de la opción asiática con promedio aritmético; ${ }^{4}$ véanse, al respecto: Turnbull and Wakeman (1991), Levy (1992), Thompson (1999), Vecer (2001), Vecer y Xu (2004), Vanmaele, et al. (2006), Wilmott (2006), Hoogland, J. and D. Neumann (2000), Fusai y Meucci (2008), E Bayraktar y Xing (2011) y Cerný y Kyriakou (2011), entre otros. Así pues Turnbull and Wakeman (1991) proporcionan un algoritmo que calcula el precio de las opciones asiáticas con promedio aritmético y lo comparan contra el método Montecarlo, mostrando ser preciso. Por su parte Vecer, (2001) proporciona un enfoque unificador para fijar precios de opciones asiáticas, con media aritmética discreta y continua. La EDP unidimensional que obtiene para el precio de la opción asiática se puede implementar relativamente fácil, incluso para casos con dividendos continuos y discretos generando además resultados rápidos y precisos.

Es pertinente observar que existe un considerable número de modelos y metodologías en la literatura financiera para obtener el precio de opciones asiáticas con promedio aritmético mediante fórmulas aproximadas. Sin embargo, el objetivo de esta investigación es proporcionar un modelo alternativo que permita caracterizar el precio de una opción asiática de venta mediante un sistema de ecuaciones diferenciales proveniente de un modelo de control óptimo estocástico en tiempo continuo, que en su diseño contemple además de los supuestos del mercado, las preferencias de los agentes económicos. Para tal efecto se desarrolla un modelo de un agente racional que dispone de una riqueza inicial y que enfrenta la decisión de cómo distribuir su riqueza entre consumo e inversión en un portafolio de activos, incluyendo una opción asiática de venta, en un horizonte de temporal finito de magnitud estocástica. Es decir, la hipótesis es que mediante equilibrio general dinámico estocástico es posible encontrar las trayectorias óptimas de consumo e inversión así como la ecuación diferencial parcial mediante la cual se valúa una opción asiática de venta de tipo europeo con precio de ejercicio flotante igual a la media aritmética.

Las características distintivas de este documento, en el marco de las investigaciones mencionadas, son: 1) La valuación del producto derivado

alcance el activo subyacente de la opción a lo largo de la vida de la opción y la volatilidad de la media siempre será inferior a la volatilidad del activo. Esto es posible verlo apartir de las griegas del Modelo de Black-Scholes, ya que se sabe que la relación entre el precio de la opción y la volatilidad del subyacente es directa.

4 Porque el precio del subyacente tiene una distribución lognormal y el promedio aritmético no tiene una distribución lognormal. 
asiático de tipo europeo se efectúa con base en la racionalidad económica, 2) La delimitación matemática del problema evita que el agente económico se endeude y/o caiga en bancarrota, y 3) La obtención de la ecuación que caracteriza el precio de la opción asiática de venta se obtiene mediante el enfoque de control óptimo estocástico en programación dinámica.

Este trabajo está organizado de la siguiente forma. En el segundo apartado se describe analíticamente el problema de control óptimo estocástico, en donde se incluye como activo de inversión, la tenencia de una opción asiática de venta con un subyacente conducido por el movimiento geométrico browniano. En el transcurso del tercer apartado se establece la restricción presupuestal intertemporal del agente representativo. En la cuarta sección se introduce el horizonte de planeación finito y de magnitud estocástica a través de un problema de control en tiempo continuo de consumo e inversión óptimos, estableciendo formalmente el problema a optimizar. En la quinta sección se proporciona una solución al problema de control óptimo planteado, mediante la cual se obtienen como resultados centrales: primero la ecuación diferencial parcial de Hamilton-Jacobi-Bellman, segundo, las condiciones de primer orden que llevan a las decisiones óptimas de las variables de control. En la sexta sección se da solución al problema de control óptimo planteado, obteniendo las proporciones óptimas de consumo e inversión. En el curso de la séptima sección se obtiene una caracterización del precio de una opción asiática de venta en términos del premio al riesgo de mercado, el cual se obtiene mediante la solución de una ecuación diferencial parcial lineal de segundo orden. En la octava sección se lleva a cabo la verificación de la solución encontrada con programación dinámica del modelo, mediante la solución a la ecuación HJB. Por último se presentan en la novena sección las conclusiones del presente trabajo indicando aquellas extensiones previstas en la agenda futura de investigación.

\section{Planteamiento del modelo de control óptimo estocástico para el problema de consumo e inversión óptimos}

Considere un agente económico racional que desea maximizar su utilidad por el consumo e inversión en un horizonte temporal de planeación finito, lo que se representa mediante el intervalo de tiempo $[0, T]$. En el tiempo $t=0$, el agente es dotado con una riqueza inicial $X_{0}$ y enfrenta el problema de cómo distribuir su riqueza entre inversión y consumo, de tal forma que su riqueza no sea negativa en su horizonte de planeación y tal que maximice su función de utilidad esperada y descontada por el consumo de un bien genérico. Así pues, se supone que la utilidad total del agente está dada por

$$
\mathrm{E}\left[\int_{0}^{T} F\left(c_{s}, s\right) \mathrm{d} s+\Phi\left(X_{t}\right) \mid \mathcal{F}_{0}\right]
$$

en donde $F$ es la función de utilidad (descontada) por el consumo, $\Phi$ es la función de retiro al tiempo $T$, la cual mide la utilidad de tener algún recurso al final del periodo, y $F_{0}$ es la información disponible al tiempo $t$.

Se supone que al agente económico le es permitido invertir sus recursos en activos con y sin riesgo, por lo que una parte de su riqueza la destina como ahorro en un banco, que paga una tasa de interés constante $r>0$, libre de 
riesgo de incumplimiento. De donde, el saldo de la inversión en el tiempo $t$ es $M_{t}=M_{0} e^{r t}$, el cual puede ser expresado mediante el problema de valor inicial

$$
\mathrm{d} M_{t}=r M_{t} \mathrm{~d} t, \quad \text { con } M_{0} \text { dado, }
$$

que implica un rendimiento

$$
\mathrm{dR}_{M} \equiv \frac{\mathrm{d} M_{t}}{M_{t}}=r \mathrm{~d} t
$$

La otra parte de su riqueza la invierte en dos activos con riesgo, a saber, una acción cuya dinámica de precios es conducida por la ecuación diferencial estocástica del movimiento geométrico Browniano,

$$
\mathrm{d} S_{t}=\mu S_{t} \mathrm{~d} t+\sigma S_{t} \mathrm{~d} W_{t}
$$

y cuyos rendimientos son representados analíticamente por la siguiente ecuación

$$
\mathrm{d} R_{S} \equiv \frac{\mathrm{d} S_{t}}{S_{t}}=\mu \mathrm{d} t+\sigma \mathrm{d} W_{t}
$$

donde $\mu \in$ y $0<\sigma \in$, son constantes que se interpretan como el rendimiento medio esperado de $S_{t}$ y su volatilidad instantánea, y $W_{t}$ es un movimiento browniano, definido sobre un espacio fijo de probabilidad con su filtración aumentada $\left(\Omega, \mathcal{F},\left(\mathcal{F}_{t}^{W}\right)_{t \in[0, T]}, P\right)$. El segundo activo es un contrato de opción asiática de venta posición larga con propósitos de cobertura y suscrito sobre la acción cuyo proceso de precios es definido por (2). Para una opción asiática de venta con precio de ejercicio variable igual a la media aritmética, el precio del contrato en la fecha de vencimiento $T$ es iguala a $\max \left(K_{t, T}-S_{T}, 0\right)$, en donde $K_{t, T}=\frac{1}{T-t} \int_{t}^{T} S_{u} \mathrm{~d} u$. Toda vez que el horizonte de planeación es el intervalo $[0 . T]$, se define,

$$
I_{t}=\int_{0}^{T} S_{u} \mathrm{~d} u \Rightarrow \mathrm{d} I_{t}=S_{t} \mathrm{dt}
$$

y dado que (2) es un proceso relativo a la filtración $\left(F_{t}^{W}\right)_{t \in[0, T]}$, entonces el precio actual es independiente del histórico precedente por lo que $I_{t}$ y $S_{T}$ pueden ser tratados de manera independiente.

Se denota entonces el precio de una opción asiática de venta, mediante $A_{t}=A_{t}\left(S_{t}, I_{t}, t\right)$; al aplicar el Lema de Itô se obtiene que,

$$
\mathrm{d} A_{t}=\left(\frac{\partial A_{t}}{\partial t}+\frac{\partial A_{t}}{\partial S_{t}} \mu S_{t}+\frac{\partial A_{t}}{\partial I_{t}} S_{t}+\frac{1}{2} \frac{\partial^{2} A_{t}}{\partial^{2} S_{t}} \sigma^{2} S_{t}^{2}\right) \mathrm{d} t+\frac{\partial A_{t}}{\partial S_{t}} \sigma S_{t} \mathrm{~d} W_{t}
$$


El rendimiento de la opción está dado por el cambio porcentual de la prima, es decir,

$$
\mathrm{d} R_{A} \equiv \frac{\mathrm{d} A_{t}}{A_{t}}
$$

Si a partir de (4) se denota a:

$$
\mu_{A}=\frac{1}{A_{t}}\left(\frac{\partial A_{t}}{\partial t}+\frac{\partial A_{t}}{\partial S_{t}} \mu S_{t}+\frac{\partial A_{t}}{\partial I_{t}} S_{t}+\frac{1}{2} \frac{\partial^{2} A_{t}}{\partial^{2} S_{t}} \sigma^{2} S_{t}^{2}\right) \mathrm{y} \sigma_{A}=\frac{1}{A_{t}}\left(\frac{\partial A_{t}}{\partial S_{t}} \sigma S_{t}\right)
$$

se sigue que

$$
\mathrm{d} A_{t}=A_{t} \mu_{A} \mathrm{~d} t+A_{t} \sigma_{A} \mathrm{~d} W_{t} .
$$

Las proporciones de riqueza que se destinarán a los activos riesgosos, la acción y la opción asiática de venta, así como al activo sin riesgo en el portafolio de inversión, en el tiempo $t$, se denotarán mediante $\theta_{1 t}, \theta_{2 t} \mathrm{y} 1-\theta_{1 t}-$ $\theta_{2 t}$, respectivamente. En lo que sigue denota la tasa de consumo, a la que se le impone la condición $c_{t} \geq 0, \forall t \geq 0$. Además, se supone que todas las estrategias de consumo e inversión son autofinanciables y se supone también que las negociaciones se llevan a cabo en forma continua (los mercados nunca cierran), sin incurrir en ningún momento en costos por comisiones a agentes de casas de bolsa ni pagos de impuestos a autoridades fiscales. Adicionalmente se supone que las ventas en corto son permitidas e ilimitadas. ${ }^{5}$

\section{Restricción presupuestal intertemporal del agente representativo}

De esta manera, si $X_{t}$ representa la riqueza del consumidor en el tiempo $t$, entonces la dinámica del proceso de la riqueza está dada por la siguiente expresión:

$$
\begin{aligned}
& \mathrm{d} X_{t}=X_{t}\left(1-\theta_{1 t}-\theta_{2 t}\right) \mathrm{d} R_{M}+X_{t} \theta_{1 t} \mathrm{~d} R_{S}+X_{t} \theta_{2 t} \mathrm{~d} R_{A}-c_{t} \mathrm{~d} t \\
& =X_{t}\left(1-\theta_{1 t}-\theta_{2 t}\right) r \mathrm{~d} t+X_{t} \theta_{1 t}\left(\mu \mathrm{d} t+\sigma \mathrm{d} W_{t}\right) \\
& +X_{t} \theta_{2 t}\left(\mu_{A} \mathrm{~d} t+\sigma_{A} \mathrm{~d} W_{t}\right)-c_{t} \mathrm{~d} t \\
& =X_{t} \theta_{1 t}(\mu-r) \mathrm{d} t+X_{t} \theta_{2 t}\left(\mu_{A}-r\right) \mathrm{d} t+\left(X_{t} r-c_{t}\right) \mathrm{d} t \\
& +X_{t} \theta_{1 t} \sigma \mathrm{d} W_{t}+X_{t} \theta_{2 t} \sigma_{A} \mathrm{~d} W_{t} \\
& =X_{t}\left(r+\theta_{1 t}(\mu-r)+\theta_{2 t}\left(\mu_{A}-r\right)-\frac{c_{t}}{X_{t}}\right) \mathrm{d} t \\
& +X_{t}\left(\theta_{1 t} \sigma+\theta_{2 t} \sigma_{A}\right) \mathrm{d} W_{t},
\end{aligned}
$$

equivalentemente,

$$
\frac{\mathrm{d} X_{t}}{X_{t}}=\mu_{X} \mathrm{~d} t+\sigma_{X} \mathrm{~d} W
$$

donde

$$
\mu_{X}=\left(r+\theta_{1 t}(\mu-r)+\theta_{2 t}\left(\mu_{A}-r\right)-\frac{c_{t}}{X_{t}}\right) \quad \text { y } \quad \sigma_{X}=\left(\theta_{1 t} \sigma+\theta_{2 t} \sigma_{A}\right) .
$$

\footnotetext{
5 Hasta donde las condiciones de frontera del problema asociado así lo permitan.
} 
Observe en la ecuación (7), que los términos $X_{t} \theta_{1 t} \mu t$ y $X_{t} \theta_{2 t} \mu_{A}$ representan el rendimiento esperado por invertir $X_{t} \theta_{1 t}$ dólares en la acción y $X_{t} \theta_{2 t}$ dólares en la opción asiática de venta durante el periodo de $t$ a $t+d t ; X_{t} \theta_{1 t} \sigma \mathrm{d} W_{t} \mathrm{y} \theta_{2 t} \sigma_{A} \mathrm{~d} W_{t}$ representan el riesgo implicado en invertir los $X_{t} \theta_{1 t}$ y $X_{t} \theta_{2 t}$ dólares en los activos riesgosos, el término $X_{t}\left(1-\theta_{1 t}-\theta_{2 t}\right) r \mathrm{~d} t$ es el interés ganado por el ahorro de $X_{t}\left(1-\theta_{1 t}-\theta_{2 t}\right)$ dólares y finalmente $c_{t} \mathrm{~d} t$ representa el consumo en el intervalo de tiempo de $t$ a $t+\mathrm{d} t$ (Sethi y Thompson 2000).

\section{Tiempo de paro y problema de control óptimo estocástico}

\subsection{Tiempo de paro}

Dados los supuestos del problema, obsérvese que el agente puede pedir prestada una cantidad ilimitada e invertirla por ejemplo en acciones, por lo que su riqueza podría llegar a ser cero en algún momento e incluso negativa, lo que corresponde matemáticamente a que el problema degenere. Para librar este problema, se restringe el dominio a $D=[0, T] \times\{x \mid x>0\}$, y se define la función

$$
\tau=\min \left[\inf \left\{t>0 \mid X_{t}=0\right\}, T\right],
$$

la interpretación correspondiente es que cuando el proceso de riqueza pegue en la frontera del dominio, es decir, sea cero, entonces la actividad se termina y por ende ya no hay herencia, de esta manera lo natural es que $\Phi$ sea cero (Björk, 2009).

\subsection{Problema de control optimo estocástico}

Ahora se establece formalmente el problema de maximización de utilidad del consumidor como un problema de control óptimo estocástico,

$$
\begin{aligned}
& \underset{\theta_{1 t}, \theta_{2 t}, c_{t}}{\operatorname{Maximizar} \mathrm{E}}\left[\int_{0}^{\tau} F\left(c_{t}, t\right) \mathrm{d} t \mid \mathcal{F}_{0}\right] \\
& \mathrm{d} X_{t}=X_{t} \mu_{X} \mathrm{~d} t+X_{t} \sigma_{X} \mathrm{~d} W_{t} \\
& X_{0}=x_{0} \\
& \theta_{1 t}+\theta_{2 t}=1 \\
& c_{t} \geq 0, \forall t \geq 0 .
\end{aligned}
$$

donde, el integrando $F\left(c_{t}, t\right)$ es la función de utilidad instantánea por el consumo. 


\section{Programación dinámica: ecuación diferencial parcial HJB}

Para dar solución al problema de (10) y encontrar las proporciones óptimas en el portafolio de inversión y el consumó óptimo del agente maximizador, se define la función de valor de la siguiente manera:

$$
\begin{aligned}
& J\left(X_{t}, I_{t}, t\right)=\max _{\theta_{1 t}, \theta_{2 t} \in R, 0 \leq\left. c_{s}\right|_{[t, \tau]}} \mathrm{E}\left[\int_{t}^{\tau} F\left(c_{s}, s\right) \mathrm{d} s \mid F_{t}\right] \\
& =\max _{\theta_{1 t}, \theta_{2 t} \in R, 0 \leq\left. c_{s}\right|_{[t, \tau]}} \mathrm{E}\left[\int_{t}^{t+\mathrm{d} t} F\left(c_{s}, s\right) \mathrm{d} s+\int_{t+\mathrm{d} t}^{\tau} F\left(c_{s}, s\right) \mathrm{d} s \mid F_{t}\right] .
\end{aligned}
$$

Después de aplicar al primer sumando el teorema del valor medio del cálculo integral y recursividad al segundo sumando, se obtiene que

$$
\begin{aligned}
J\left(X_{t}, I_{t}, t\right) & =\max _{\theta_{1 t}, \theta_{2 t} \in R, 0 \leq\left. c_{t}\right|_{[t, t+\mathrm{d} t]}} \mathrm{E}\left\{F\left(c_{t}, t\right) \mathrm{d} t+o(\mathrm{~d} t)\right. \\
& \left.+J\left(X_{t}+\mathrm{d} X_{t}, I_{t}+\mathrm{d} I_{t}, t+\mathrm{d} t\right) \mid \mathcal{F}_{t}\right\}
\end{aligned}
$$

Al emplear la expansión en serie de Taylor al segundo sumando, se tiene

$$
0=\max _{\theta_{1 t}, \theta_{2 t} \in R, 0 \leq\left. c_{s}\right|_{[t, t+\mathrm{d} t]}} \mathrm{E}\left\{F\left(c_{t}, t\right) \mathrm{d} t+o(\mathrm{~d} t)+\mathrm{d} J\left(X_{t}, I_{t}, t\right) \mid \mathcal{F}_{t}\right\} .
$$

simplificando,

$$
0=\max _{\theta_{1 t}, \theta_{2 t} \in R, 0 \leq\left. c_{s}\right|_{[t, t+\mathrm{d} t]}} \mathrm{E}\left\{F\left(c_{t}, t\right) \mathrm{d} t+o(\mathrm{~d} t)+\mathrm{d} J\left(X_{t}, I_{t}, t\right) \mid \mathcal{F}_{t}\right\}
$$

$\mathrm{Al}$ aplicar a d $J\left(X_{t}, I_{t}, t\right)$ el lema de Itô y después de simplificar, se tiene

$$
\begin{aligned}
0 & =\max _{\theta_{1 t}, \theta_{2 t} \in R, 0 \leq\left. c_{t}\right|_{[t, t+\mathrm{d} t]}} \mathrm{E}\left\{F\left(c_{t}, t\right) \mathrm{d} t+o(\mathrm{~d} t)+\frac{\partial J\left(X_{t}, I_{t}, t\right)}{\partial X_{t}} X_{t} \sigma_{X} \mathrm{~d} W_{t}\right. \\
& \left.+\left[\frac{\partial J\left(X_{t}, I_{t}, t\right)}{\partial t}+\frac{\partial J\left(X_{t}, I_{t}, t\right)}{\partial I_{t}} S_{t}+\frac{\partial J\left(X_{t}, I_{t}, t\right)}{\partial X_{t}} X_{t} \mu_{X}+\right] \mathrm{d} t \mid \mathcal{F}_{t}\right\}
\end{aligned}
$$

A continuación se obtiene el valor esperado de esta última ecuación, y dado que $d W_{t}$ se distribuye $N(0, \mathrm{~d} t)$, se elimina el término con browniano, de lo que resulta

$$
\begin{aligned}
& 0=\max _{\theta_{1 t}, \theta_{2 t} \in R, 0 \leq\left. c_{t}\right|_{[t, t+\mathrm{d} t]}}\left\{F\left(c_{t}, t\right) \mathrm{d} t+o(\mathrm{~d} t)+\frac{1}{2} \frac{\partial^{2} J\left(X_{t}, I_{t}, t\right)}{\partial X_{t}^{2}} X_{t}^{2} \sigma_{X}^{2} \mathrm{~d} t\right. \\
& \left.+\frac{\partial J\left(X_{t}, I_{t}, t\right)}{\partial I_{t}} S_{t} \mathrm{~d} t+\frac{\partial J\left(X_{t}, I_{t}, t\right)}{\partial t} \mathrm{~d} t+\frac{\partial J\left(X_{t}, I_{t}, t\right)}{\partial X_{t}} X_{t} \mu_{X} \mathrm{~d} t \mid \mathcal{F}_{t}\right\} .
\end{aligned}
$$


Ahora se divide la expresión anterior entre $d t$ y se toma el límite de la misma cuando $\mathrm{d} t \rightarrow 0$

$$
\begin{aligned}
& 0=\max _{\theta_{1 t}, \theta_{2 t} \in R, 0 \leq c_{t}}\left\{F\left(c_{t}, t\right)+\frac{\partial J\left(X_{t}, I_{t}, t\right)}{\partial t}+\frac{\partial J\left(X_{t}, I_{t}, t\right)}{\partial X_{t}} X_{t} \mu_{X}\right. \\
& \left.+\frac{\partial J\left(X_{t}, I_{t}, t\right)}{\partial I_{t}} S_{t}+\frac{1}{2} \frac{\partial^{2} J\left(X_{t}, I_{t}, t\right)}{\partial X_{t}^{2}} X_{t}^{2} \sigma_{X}^{2}\right\} .
\end{aligned}
$$

A la última ecuación anexamos las condiciones de frontera correspondientes, para obtener la EDP de HJB

$$
\left\{\begin{array}{l}
0=\max _{\theta_{1 t}, \theta_{2 t} \in R, 0 \leq\left. c_{t}\right|_{[t, t+\mathrm{d} t]}}\left\{F\left(c_{t}, t\right)+\frac{\partial J\left(X_{t}, I_{t}, t\right)}{\partial t}+\frac{\partial J\left(X_{t}, I_{t}, t\right)}{\partial X_{t}} X_{t} \mu_{X}\right. \\
\left.+\frac{\partial J\left(X_{t}, I_{t}, t\right)}{\partial I_{t}} S_{t}+\frac{1}{2} \frac{\partial^{2} J\left(X_{t}, I_{t}, t\right)}{\partial X_{t}^{2}} X_{t}^{2} \sigma_{X}^{2}\right\} \\
J\left(X_{t}, I_{t}, T\right)=0 \\
J\left(0, I_{t}, t,\right)=0
\end{array}\right.
$$

\subsection{Función de utilidad}

Ahora se usará una función de utilidad de la forma $F\left(c_{t}, t\right)=e^{-\rho t} V\left(c_{t}\right)$, donde $V\left(c_{t}\right)$ es un miembro de la familia de funciones de utilidad HARA (Merton, 1990 y Hakansson, 1970), particularmente, se elige la función de consumo

$$
F\left(c_{t}, t\right)=e^{-\rho t} c^{\gamma}, \quad 0<\gamma<1 .
$$

Observe que $V\left(c_{t}\right)$ tiene la propiedad de que

$$
V^{\prime}(0)=\left.\gamma \frac{c^{\gamma}}{c}\right|_{c=0}=\infty,
$$

lo que forzará que el consumo sea positivo a través del horizonte de planeación.

\subsection{Condiciones de primer orden}

Al suponer máximo interior y hacer las sustituciones correspondientes en la EDP de HJB, se tiene

$$
\begin{aligned}
& 0=e^{-\rho t} c^{\gamma}+\frac{\partial J\left(X_{t}, I_{t}, t\right)}{\partial t}+\frac{1}{2} \frac{\partial^{2} J\left(X_{t}, I_{t}, t\right)}{\partial X_{t}^{2}} X_{t}^{2}\left(\theta_{1 t} \sigma+\theta_{2 t} \sigma_{A}\right)^{2}+ \\
& +\frac{\partial J\left(X_{t}, I_{t}, t\right)}{\partial I_{t}} S_{t}+\frac{\partial J\left(X_{t}, I_{t}, t\right)}{\partial X_{t}} X_{t}\left(r+\theta_{1 t}(\mu-r)+\theta_{2 t}\left(\mu_{A}-r\right)-\frac{c_{t}}{X_{t}}\right)
\end{aligned}
$$

Ahora, lo que se requiere es optimizar para $\theta_{1 t}, \theta_{2 t}$ y $c_{t}$, de donde se obtienen las condiciones de primer orden,

$$
c_{t}=\left[\frac{\partial J\left(X_{t}, I_{t}, t\right)}{\partial X_{t}} \frac{e^{\rho t}}{\gamma}\right]^{\frac{1}{\gamma-1}},
$$




$$
\begin{gathered}
\theta_{1 t}=-\frac{\frac{\partial J\left(X_{t}, I_{t}, t\right)}{\partial X_{t}} X_{t}(\mu-r)+\frac{\partial^{2} J\left(X_{t}, I_{t}, t\right)}{\partial X_{t}^{2}} X_{t}^{2} \sigma \theta_{2 t} \sigma_{A}}{\frac{\partial^{2} J\left(X_{t}, I_{t}, t\right)}{\partial X_{t}^{2}} X_{t}^{2} \sigma^{2}}, \\
\theta_{2 t}=-\frac{\frac{\partial J\left(X_{t}, I_{t}, t\right)}{\partial X_{t}} X_{t}\left(\mu_{A}-r\right)+\frac{\partial^{2} J\left(X_{t}, I_{t}, t\right)}{\partial X_{t}^{2}} X_{t}^{2} \sigma \theta_{1 t} \sigma_{A}}{\frac{\partial^{2} J\left(X_{t}, I_{t}, t\right)}{\partial X_{t}^{2}} X_{t}^{2} \sigma_{A}^{2}} .
\end{gathered}
$$

\section{Solución al problema de control: decisiones óptimas}

Para elegir la función $J\left(X_{t}, I_{t}, t\right)$, que satisfaga la EDP de HJB y toda vez que se trata de una ecuación diferencial parcial no lineal, su solución es un producto de funciones separables, de tal forma que:

$$
J\left(X_{t}, I_{t}, t\right)=e^{-\rho t} h(t) X_{t}^{\gamma},
$$

junto con $h(T)=0$ debido a las condiciones de frontera de la ecuación HJB. Dado $J$, se tiene,

$$
\begin{aligned}
& \frac{\partial J\left(X_{t}, I_{t}, t\right)}{\partial t}=X_{t}^{\gamma} e^{-\rho t} \frac{\partial h\left(I_{t}, t\right)}{\partial t}-\rho X_{t}^{\gamma} e^{-\rho t} h\left(I_{t}, t\right) \\
& \frac{\partial J\left(X_{t}, I_{t}, t\right)}{\partial I_{t}}=X_{t}^{\gamma} e^{-\rho t} \frac{\partial h\left(I_{t}, t\right)}{\partial I_{t}} \\
& \frac{\partial J\left(X_{t}, I_{t}, t\right)}{\partial X_{t}}=\gamma X_{t}^{\gamma-1} e^{-\rho t} h\left(I_{t}, t\right) \\
& \frac{\partial^{2} J\left(X_{t}, I_{t}, t\right)}{\partial X_{t}^{2}}=\gamma(\gamma-1) X_{t}^{\gamma-2} e^{-\rho t} h\left(I_{t}, t\right) .
\end{aligned}
$$

Si sustituimos los valores de (18) en (14), (15) y (16), se obtiene:

$$
\begin{gathered}
\hat{c}_{t}=X_{t} h^{\frac{1}{\gamma-1}}(t), \\
\hat{\theta}_{1 t}=-\frac{(\mu-r)+(\gamma-1) \sigma \theta_{2 t} \sigma_{A}}{(\gamma-1) \sigma^{2}}, \\
\hat{\theta}_{2 t}=-\frac{\left(\mu_{A}-r\right)+(\gamma-1) \sigma \theta_{1 t} \sigma_{A}}{(\gamma-1) \sigma_{A}^{2}} .
\end{gathered}
$$

Es pertinente señalar que $\hat{c}$ es lineal y estocástica en la riqueza, lo que es propio del entorno contingente en el que se desarrolla el modelo y de la realidad incierta de los mercados económicos y financieros, mientras que las proporciones óptimas $\hat{\theta}_{1 t}$ y $\hat{\theta}_{2 t}$ forman un sistema redundante de ecuaciones lineales, a saber,

$$
\left\{\begin{array}{l}
\hat{\theta}_{1 t}+\frac{\hat{\theta}_{2 t} \sigma_{A}}{\sigma}=-\frac{\mu-r}{(\gamma-1) \sigma^{2}} \\
\hat{\theta}_{2 t}+\frac{\hat{\theta}_{1 t} \sigma}{\sigma_{A}}=-\frac{\mu_{A}-r}{(\gamma-1) \sigma_{A}^{2}}
\end{array}\right.
$$

Dado por, $\lambda=\frac{\mu-r}{(1-\gamma) \sigma^{2}}$ y $\lambda_{A}=\frac{\mu_{A}-r}{(1-\gamma) \sigma_{A}^{2}}$, que representan los premios al riesgo de mercado de la acción y de la opción asiática de venta, respectivamente, y 
que como se verá coinciden proporcionalmente. Al considerar, $\xi=\frac{\sigma_{A}}{\sigma}$ se tiene, se tiene:

$$
\left.\begin{array}{c}
\hat{\theta}_{1 t}+\xi \hat{\theta}_{2 t}=\lambda, \\
\frac{\hat{\theta}_{1 t}}{\xi}+\hat{\theta}_{2 t}=\lambda_{A},
\end{array}\right\} \Rightarrow \underbrace{\left(\begin{array}{cc}
1 & \xi \\
\xi^{-1} & 1
\end{array}\right)}_{D}\left(\begin{array}{c}
\hat{\theta}_{1 t} \\
\hat{\theta}_{2 t}
\end{array}\right)=\left(\begin{array}{c}
\lambda \\
\lambda_{A}
\end{array}\right) .
$$

A partir de (23) observe que $\operatorname{det}(D)=0$, es decir, el sistema de ecuaciones tiene una infinidad de soluciones, equivalentemente decimos que los premios al riesgo de mercado para la acción y para opción asiática de venta son combinación lineal uno del otro, y esto sucede porque la opción hereda propiedades del proceso de precios del subyacente.

\section{Premio al riesgo de mercado de una opción asiática de venta de tipo europeo}

A partir del sistema de ecuaciones (23) se tiene que,

$$
\lambda=\xi \lambda_{A}
$$

es decir, los premios al riesgo del mercado del subyacente y de la opción son equivalentes,

$$
\text { ie) } \frac{\mu-r}{(1-\gamma) \sigma^{2}}=\frac{\sigma_{A}}{\sigma} \frac{\mu_{A}-r}{(1-\gamma) \sigma_{A}^{2}},
$$

lo cual conduce

$$
(\mu-r) \frac{\sigma_{A}}{\sigma}=\left(\mu_{A}-r\right) \text {. }
$$

Ahora se realizan las sustituciones de $\mu_{A}$ y $\sigma_{A}$ que se definen a partir de (14), de donde se obtiene

$$
\frac{\partial A_{t}}{\partial t}+\frac{\partial A_{t}}{\partial I_{t}} S_{t}+\frac{1}{2} \frac{\partial^{2} A_{t}}{\partial^{2} S_{t}} \sigma^{2} S_{t}^{2}+\frac{\partial A_{t}}{\partial S_{t}} S_{t} r-r A_{t}=0
$$

(24) es una ecuación diferencial parcial de segundo orden mediante la cual se valuará la opción asiática de venta; como es bien sabido tiene una familia infinita de curvas solución por lo que para poder hacer la valuación correspondiente a la opción asiática, se debe de imponer a esta una condición final, que corresponde a la fecha de término del contrato. Esto es,

$$
\left\{\begin{array}{l}
\frac{\partial A_{t}}{\partial t}+\frac{\partial A_{t}}{\partial I_{t}} S_{t}+\frac{1}{2} \frac{\partial^{2} A_{t}}{\partial^{2} S_{t}} \sigma^{2} S_{t}^{2}+\frac{\partial A_{t}}{\partial S_{t}} S_{t} r-r A_{t}=0 \\
\mathrm{~A}\left(S_{t}, I_{t}, T\right)=\max \left(\frac{I_{t}}{T}-S_{T}, 0\right) .
\end{array}\right.
$$

\section{Verificación de la programación dinámica: solución de la ecuación HJB}

Para dar solución al problema de control óptimo planteado, se requiere ahora encontrar la función $h(t)$ definida en $J\left(X_{t} I_{t}, t\right)$ que resuelve la ecuación HJB; para tal efecto se observa que la regla óptima de consumo es lineal en la riqueza y se supone una solución de esquina para las proporciones óptimas asignadas a la tenencia de la acción y a su opción asiática de venta, de tal forma que $\hat{\theta}_{1 t}=0$ y $\hat{\theta}_{2 t}=1$. Se ha elegido esta solución toda vez que las ventas en corto son permitidas. Si se sustituyen en la ecuación (13) los valores de la ecuación 
(19), (20), (21), $\hat{c}_{t}, \mu_{A}, \sigma_{A} \hat{\theta}_{1 t}=0$ y $\hat{\theta}_{2 t}=1$, y las derivadas parciales en $\mu_{A} \mathrm{y}$ $\sigma_{A}$ se evalúan en el dinero, es decir, $S_{t}=K_{t, T}$, las cuales se denotan mediante

$$
\left.\mu_{A}\right|_{S_{t}=K_{t, T}}=\left.\bar{\mu}_{A} \quad \mathrm{y} \quad \sigma_{A}\right|_{S_{t}=K_{t, T}}=\bar{\sigma}_{A}
$$

se obtiene:

$$
0=X_{t}^{\gamma}\left\{h^{\prime}(t)+h(t)\left((-\rho)+\gamma \bar{\mu}_{A}+\frac{\gamma}{2}(\gamma-1) \bar{\sigma}_{A}^{2}\right)+h^{\frac{\gamma}{\gamma-1}}(t)\right\} .
$$

junto con la condición de frontera $h(I, T)=0$.

Observe que la solución de esta ecuación diferencial no depende de $I_{t}$. Si además, se denota mediante $k_{1}=(-\rho)+\gamma \bar{\mu}_{A}+\frac{\gamma}{2}(\gamma-1) \bar{\sigma}_{A}^{2}$ a el coeficiente de $h_{t}$, y se simplifica, tiene la ecuación diferencial ordinaria de primer orden,

$$
h^{\prime}(t)+k_{1} h(t)=-(\gamma-1) h^{\frac{\gamma}{\gamma-1}}(t),
$$

Si esta ecuación se cumple para todas $t$, entonces $h_{t}$ debe de resolver la ecuación diferencial

$$
h^{\prime}(t)+k_{1} h(t)=-h^{\frac{\gamma}{\gamma-1}}(t), \quad h(T)=0,
$$

la cual es una ecuación diferencial de primer orden tipo Bernoulli donde: $p(t)=$ $k_{1}, q(t)=-1$ y $n=\frac{\gamma}{\gamma-1}$. Para transformar la ecuación de Bernoulli en una ecuación diferencial ordinaria lineal de primer orden de una función (desconocida), se sustituye $z=h^{1-n}(t)=h^{-\frac{1}{\gamma-1}}(t)$, de donde se tiene que $h(t)=z^{1-\gamma}$ y $h^{\prime}(t)=(1-\gamma) z^{-\gamma} z^{\prime}$, al sustituir en (33) y multiplicar ambos lados de la ecuación por $\frac{z^{\gamma}}{(1-\gamma)}$, se obtiene,

$$
z^{\prime}+\frac{k_{1}}{(1-\gamma)} z=-\frac{1}{(1-\gamma)},
$$

a (29) debe imponerse la condición $z(T)=0$. Así (29) es un problema de valor inicial y se resuelve mediante el método de factor integrante (Leibinitz).

$$
\mu(t)=e^{\int \frac{k_{1}}{(1-\gamma)} \mathrm{d} t}=e^{\frac{k_{1}}{(1-\gamma)} t}
$$

de donde se obtiene que $z_{t}$ es

$$
\begin{aligned}
z(t) & =\frac{\int 1 e^{\frac{k_{1} t}{1-\gamma}} \mathrm{d} t+k_{2}}{e^{\frac{k_{1} t}{1-\gamma}}} \\
& =\frac{\left(\frac{1-\gamma}{k_{1}}\right) \int e^{u} d u+k_{2}}{e^{\frac{k_{1} t}{1-\gamma}}} \\
& =\frac{\left(\frac{1-\gamma}{k_{1}}\right) e^{\frac{k_{1} t}{1-\gamma}}+k_{2}}{e^{\frac{k_{1} t}{1-\gamma}}} \\
& =\frac{1-\gamma}{k_{1}}+k_{2} e^{-\frac{k_{1} t}{1-\gamma}}
\end{aligned}
$$


ahora se impondrá a $z$ la condición inicial $z(T)=0$, de donde

$$
0=z(T)=\frac{1-\gamma}{k_{1}}+k_{2} e^{-\frac{k_{1} T}{1-\gamma}} \Rightarrow-\frac{1-\gamma}{k_{1}} e^{\frac{k_{1} T}{1-\gamma}}=k_{2}
$$

Por lo que

$$
z(t)=\frac{1-\gamma}{k_{1}}\left(1-e^{\frac{k_{1}}{1-\gamma}(T-t)}\right) .
$$

Por último, si se sustituye $z(t)$ en $h(t)$, se tiene que $h(t)=z^{1-\gamma}$ y $h^{\prime}(t)=$ $(1-\gamma) z^{-\gamma} z^{\prime}$

$$
h(t)=z^{1-\gamma}(t)=\left[\frac{1-\gamma}{k_{1}}\left(1-e^{\frac{k_{1}}{1-\gamma}(T-t)}\right)\right]^{1-\gamma}, h(T)=0 .
$$

Se ha así mostrado que si $J$ está dada por (17) con (33) definida como la solución de (28) y si definimos $\hat{\theta}_{1 t}, \hat{\theta}_{2 t}$ y $\hat{c}$ por (19), (20) y (21) entonces $J$ satisface la ecuación de HJB y $\hat{\theta}_{1 t}, \hat{\theta}_{2 t}$ y $\hat{c}$ consiguen optimizar el problema de control óptimo con horizonte temporal de magnitud estocástica.

\section{Conclusiones}

El principal objetivo de esta investigación fue el de desarrollar un modelo de equilibrio general dinámico estocástico a partir del cual fuera posible llevar a cabo la valuación de las opciones asiáticas de tipo europeo, toda vez que por su estructura y consistencia interna, la teoría de control permitiría obtener dicha valuación formulada a partir de supuestos de racionalidad económica; dotando con esto al agente económico consumidor-inversionista de herramientas para su toma de decisiones de consumo e inversión en un portafolio de activos cuando el horizonte temporal del que dispone es finito con fecha final aleatoria.

Es pertinente reconocer la importancia conjugada de la matemática y la ingeniería financiera en la modelación de fenómenos económicos. Particularmente nos referimos a la teoría de control óptimo estocástico en tiempo continuo, la cual se ha revelado como un instrumento fundamental cuando se requiere modelar alguna actividad económica que se desarrolla de manera dinámica. Dada la modelación de los supuestos financieros mediante ecuaciones diferenciales estocásticas de movimientos geométricos brownianos no correlacionados, aunado al supuesto de que la función de utilidad es del tipo $U(c, t)=e^{-\delta t} V(c)$, en donde $U$ es un miembro de la familia de funciones de tipo HARA (Hyperbolic Absolute Risk Aversion), fue posible obtener soluciones analíticas y cerradas. ${ }^{6}$

Como resultado de este modelo se plantea en la agenda de investigación futura, modelar la caracterización del precio de las opciones asiáticas de tipo

\footnotetext{
6 La principal dificultad de los problemas de control óptimo estocástico consiste en resolver la ecuación de HJB, dado que no hay disponible una teoría general para ello. No obstante, es posible encontrar soluciones analíticas y cerradas de dicha ecuación siempre que se incorpore en los supuestos del modelo que la dinámica de los precios es modelada mediante la ecuación diferencial del movimiento geométrico browniano y que la función de utilidad es del tipo HARA (Hyperbolic Absolute Risk Aversion).
} 
402 Nueva Época REMEF (The Mexican Journal of Economics and Finance)

europeo y americano implementando nuevas formas funcionales para el precio del activo subyacente que contemple los cambios imprevistos en volatilidad y en tasa de interés, ya que es esencial considerar ambientes estocásticos más ricos, porque la presencia de incertidumbre puede conducir a cambios cualitativamente significativos en los efectos de política y en la elección resultante de decisiones óptimas. 


\section{Bibliografía}

Björk, Tomas (2009). Arbitrage Theory in Continuous time. Third Edition, Oxford Finance Series.

Broadie M. \& Detemple, J. (2004). Option Pricing: Valuation Models and Applications. Management Science, Vol. 50, No. 9, pp. 1145-1177.

Bayraktar, E. \& H. Xing, (2011). Pricing Asian options for jump diffusion, Mathematical Finance, 2011 - Wiley Online Library.

Cerný, A. and I., Kyriakou, (2011). An Improved Convolution Algorithm for Discretely Sampled Asian Options. Quantitative Finance, 2011, 11(3), pp. 381-389

Cox, J. C., J. E. Ingersoll, and S. A. Ross (1985a). An Intertemporal General Equilibrium Model of Asset Prices. Econometrica, Vol. 53, No. 2, pp. 385-408.

Cox, J. C., J. E. Ingersoll, and S. A. Ross (1985b). A Theory of the Term Structure of Interest Rates. Econometrica, Vol. 53, No. 2, pp. 363-384.

Cruz-Ake, S. \& Venegas-Martínez, F. (2010). Productos derivados sobre bienes de consumo. Econo Quantum, Vol. 6. No. 1, pp.25-54, Zapopan, México.

Fusai, G. \& A. Meucci, (2008), Pricing discretely monitored Asian options under Lévy processes, Journal of Banking 85 Finance, 32 (2008), pp. 2076-2088.

Grinols, E.R., and Turnovsky S. J. (1993). Risk, the Financial Market and Macroeconomic Equilibrium. Journal of Economic Dynamics and Control, Vol. 17, pp.1-36.

Hakansson, N. (1970). Optimal Investment and Consumption Strategies under Risk for a Class of Utility Functions.Econometrica, Vol. 38, No. 5, pp. 587-607.

Hernández-Lerma, O. (1994). Lectures on Continuous-Time Markov Control Processes. Aportaciones Matemáticas 3, Sociedad Matemática Mexicana.

Hoogland, J. \& Neumann, D. (2000). Asians and Cash Dividends: Exploiting Symmetries in Pricing Theory, Working paper, June 2000.

Hull, J. (2014). Options, Futures, and Other Derivatives, ninth edition, Pearson Education.

Huyên, P. (2009). Continuous-time Stochastic Control and Optimization with Financial Applications. Springer-Verlag, Berlin Heidelberg 2009.

Lehoczky, J., Sethi, S. \& Shreve, S. (1983), Optimal Consumption and Investment Policies Allowing Consumption Constraints and Bankruptcy. Mathematics of Operations Research, Vol. 8, No. 4 (Nov., 1983), pp. 613-636.

Martínez-Palacios, T., Martínez-Sánchez J. F. \& Venegas-Martínez, F., (2015). Consumption and portfolio decisions of a rational agent that has access to an American put option on an underlying asset with stochastic volatility. International Journal on Pure and Applied Mathematics.

Martínez-Palacios, T. \& Venegas-Martínez, F. (2014). Un modelo macroeconómico con agentes de vida finita y estocástica: cobertura de riesgo de mercado con derivados americanos. Economía: Teoría y Práctica, No. 41, semestre julio-diciembre de 2014, pp. $71-106$.

Martínez-Palacios, T.; Sánchez-Daza, A. \& Venegas-Martínez, F., (2012). Valuación de opciones americanas: un enfoque de control óptimo estocástico en un horizonte finito con fecha final aleatoria. Análisis Económico, Vol. XXVII, No. 64, primer cuatrimestre de 2012, pp. 165-183.

Merton, R. C. (1971). Optimum Consumption and Portfolio Rules in a Continuous- Time Model. Journal of Economic Theory, Vol. 3, No. 4, pp.373-413.

Merton, R. C. (1992). Continuous-Time Finance. Review of economics and statistics, Vol. 51, No. 2, pp. 247-257.

Sethi, S. \& Thompson, G. (2000). Optimal Control Theory. New York, Springer.

Sierra, G. (2007). Procesos Hurst y movimientos brownianos fraccionales en mercados fractales. Revista de administración finanzas y economía, Vol. 1, No. 1, 2007, pp. 1-21.

Tavella, D. \& C. Randall, (2000). Pricing Financial Instuments: The Finite Difference Method, Wiley 2000.

Thompson, G. (1999), Fast narrow bounds on the value of Asian options, Working paper.

Turnbull, S., Wakeman, L. (1992). A Quick Algorithm for Pricing European average Options. Journal of Financial and Quantitative Analysis, 26, pp. 377389. 
404 Nueva Época REMEF (The Mexican Journal of Economics and Finance)

Turnovsky, S. J. (1993). Macroeconomic Policies, Growth, and Welfare in a Stochastic Economy. International Economic Review, No. 13, pp. 183-212.

Turnovsky S. \& Smith W. (2006). Equilibrium Consumption and Precautionary Savings in a Stochastically Growing Economy. Journal of Economic Dynamics \& Control, Vol. 30, pp. 243278.

Vanmaele M., G. Deelstra, J. Liinev, J. Dhaene, \& M. Goovaerts, (2006). Bounds for the Price of Discrete Arithmetic Asian Options Journal of Computational and Applied Mathematics, vol. 185, No. 1, 1 January 2006, pp. 51-90.

Vecer, J., (2000). A New PDE Approach for Pricing Arithmetic average Asian Options, The Journal of Computational Finance, January 2000.

Vecer, J. y M. Xu, (2004). Pricing Asian Options in a Semimartingale Model, Quantitative Finance, 2004.

Venegas-Martínez, F. (2006). Stochastic Temporary Stabilization: Undiversifiable Devaluation and Income Risks. Economic Modelling, Vol. 23, No. 1, pp. 157-173.

Venegas-Martínez, F. (2007). Real Options on Consumption in a Small Open Monetary conomy: A Stochastic Optimal Control Approach. Morfismos, Vol. 11. No. 1, pp.3752.

Venegas-Martínez F. y Ortiz-Arango F. (2010). Impacto de la política fiscal en un ambiente con inflación estocástica: un modelo de control óptimo. Morfismos, Vol. 14, No.1, pp. 51-68.

Wilmott, P. (2006). On quantitative finance. Second edition, England, John Wiley \& Sons Ltd. 